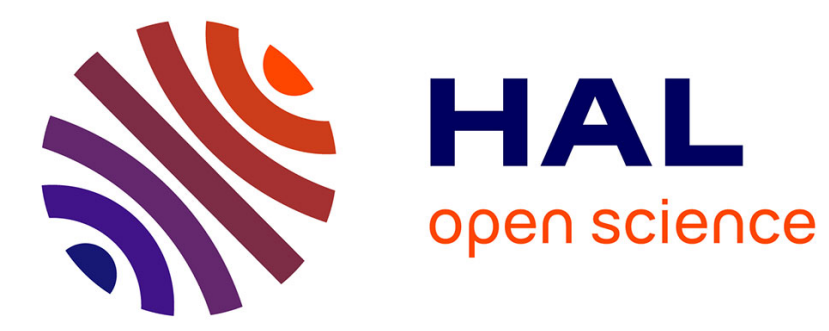

\title{
Cross-over in polymer solutions
}

B. Farnoux, F. Boue, J.P. Cotton, M. Daoud, G. Jannink, M. Nierlich, P.-G. de Gennes

\section{To cite this version:}

B. Farnoux, F. Boue, J.P. Cotton, M. Daoud, G. Jannink, et al.. Cross-over in polymer solutions. Journal de Physique, 1978, 39 (1), pp.77-86. 10.1051/jphys:0197800390107700 . jpa-00208741

\section{HAL Id: jpa-00208741 https://hal.science/jpa-00208741}

Submitted on 1 Jan 1978

HAL is a multi-disciplinary open access archive for the deposit and dissemination of scientific research documents, whether they are published or not. The documents may come from teaching and research institutions in France or abroad, or from public or private research centers.
L'archive ouverte pluridisciplinaire HAL, est destinée au dépôt et à la diffusion de documents scientifiques de niveau recherche, publiés ou non, émanant des établissements d'enseignement et de recherche français ou étrangers, des laboratoires publics ou privés. 


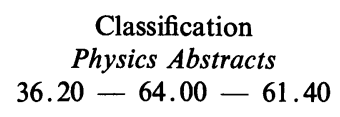

\title{
CROSS-OVER IN POLYMER SOLUTIONS
}

\author{
B. FARNOUX, F. BOUÉ, J. P. COTTON, M. DAOUD, G. JANNINK, M. NIERLICH \\ and
}

P. G. DE GENNES $(*)$

\author{
DPh-G/PSRM, CEN Saclay, Boîte Postale' no 2, 91190 Gif sur Yvette, France
}

(Reçu le 18 avril 1977, révisé le 21 juillet 1977, accepté le 8 septembre 1977)

\begin{abstract}
Résumé. - La fonction de corrélation de paire $P(r)$ pour les polymères en solution a été mesurée par diffusion de neutrons aux petits angles dans l'intervalle $3 R_{\mathrm{G}} \geqslant r \geqslant l$ où $R_{\mathrm{G}}$ est le rayon de giration et $l$ la longueur du monomère. A la température thêta cette fonction est décrite par la loi de Debye $1 / r$. En bon solvant (limite haute température) et à la limite de la concentration nulle, S. F. Edwards prédit que cette fonction est uniformément proportionnelle à $r^{-4 / 3}$.

Cependant le résultat expérimental montre que pour des concentrations assez élevées ou pour des températures intermédiaires la fonction $P(r)$ présente les deux comportements. On trouve qu'ils sont séparés par des longueurs de cross-over $r^{*}$ qui dépendent de la température et de la concentration. Le scaling de $r^{*}$ est relié au scaling de la longueur de corrélation $\xi$ et du rayon $R_{\mathrm{G}}$ dans le diagramme température-concentration.
\end{abstract}

\begin{abstract}
Using a small-angle neutron scattering experiment, we measured the pair correlation function $P(r)$ in polymer solutions in the interval $3 R_{\mathrm{G}} \geqslant r \geqslant l$, where $R_{\mathrm{G}}$ is the radius of gyration and $l$ the step length. At the theta temperature, this function is known to follow the characteristic Debye law $P(r) \sim r^{-1}$. In good solvents (high temperature limit) and in the limit of zero polymer concentration this function is uniformly proportional to $r^{-4 / 3}$, as predicted by S. F. Edwards.

We observe however, that at higher concentrations or intermediate temperatures, $P(r)$ exhibits both characteristic behaviours, depending on the range of $r$. The cross-over distances $r^{*}$ which separate the patterns are found to depend upon concentration and temperature. The scaling of $r^{*}$ is related to the scaling of the screening length $\xi$ and the radius $R_{\mathrm{G}}$ in the temperature-concentration diagram.
\end{abstract}

1. Introduction. - $\bar{W}$ e examine the monomermonomer pair correlation function of flexible polymer coils dispersed in a solvent. The statistics of such coils is often given in terms of the step length $l$ and the average squared radius of gyration $\left\langle R_{\mathrm{G}}^{2}\right\rangle$. However, in the reciprocal space defined by the scattering vector

$$
|\mathbf{q}|=(4 \pi / \lambda) \sin \frac{\theta}{2}
$$

where $\lambda$ is the radiation wavelength and $\theta$ the scattering angle, there is a domain in between the Guinier range $q R_{\mathrm{G}}<1$ and the submonomer range $q l>1$ which is very appropriate for the investigation of polymer statistics. In this intermediate range

$$
3 / R_{\mathrm{G}} \leqslant q \leqslant 1 / l
$$

(*) Collège de France, Paris. scattering experiments reveal the asymptotic behaviour of the pair correlation function in the limit of infinite molecular weight. In this range universal laws can be considered, in contrast to the more detailed [1] information obtained from diffraction patterns, which is specific to each polymer species.

Earlier results were reported concerning these laws [2,3]. Recent neutron scattering experiments have, however, brought new evidence. We therefore find it appropriate to give here a general survey of these results. The observation which we wish to discuss is the cross-over or change of behaviour. A well-known example of cross-over in polymer solutions is found in the scaling of $\left\langle R_{G}^{2}\right\rangle$ with molecular weight $M$. For the isolated coil in a good solvent, theory $[4,5]$ predicts that the coil is swollen with respect to the random walk configuration when $M$ is sufficiently great. Below a given value $M^{*}$, the coil returns to a configuration which has the essential 
observable characteristics of a random walk. The vicinity of $M^{*}$ is characterized by a change of behaviour of the coil configuration.

The space $M, T, C$ (Fig. 1 ), where $T$ is the temperature and $C$ the monomer concentration $\left(\mathrm{g} \mathrm{cm}^{-3}\right)$ is in fact partitioned into regions in which $R_{\mathrm{G}}^{2}$ has characteristic scaling laws in these three variables (Table I) (Refs. [6, 30]). As one goes from one region to another, the scaling law changes smoothly from one pattern to another. There are thus several crossovers in the polymer solution diagram [7]. Although the change of behaviour in the cross-over region was acknowledged in the literature [29], it was never studied as such. There are two reasons why a special interest in this problem is being developed at the present time.

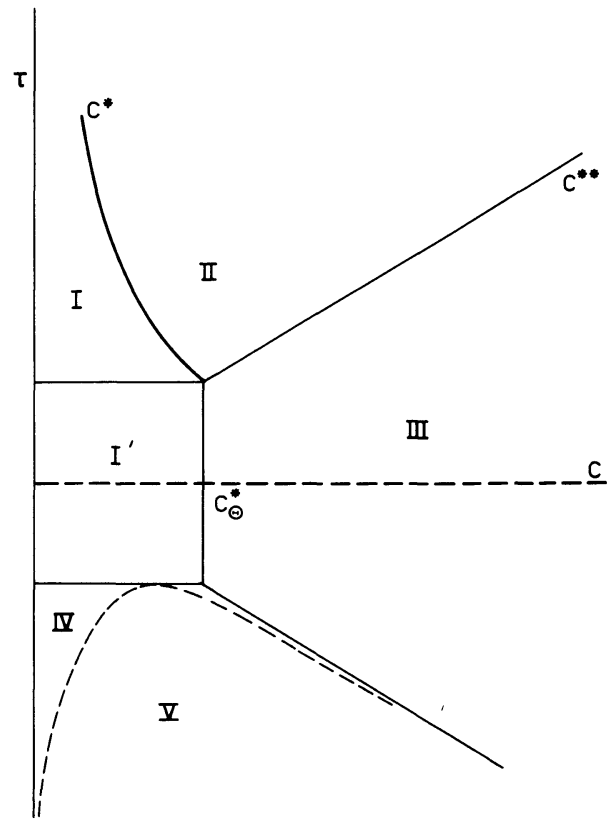

FIG. 1. - Temperature concentration diagram for flexible polymer solutions. $\tau=\frac{T-\theta}{\theta}$ is the reduced temperature and $C$ the monomer concentration. This diagram is partitioned into different regions; region I' is the Flory's theta or tricritical range. By increasing the temperature there is a cross-over to the region I, the dilute or critical range. The cross-over line is given by equation (2.6). Region II is the semi-dilute (critical) range and $C^{*}$ is the crossover line (eq. (3.2)). Region III is a tricritical domain (theta semidilute regime) limited by lines $C^{* *} \sim|\tau|$ symmetric with respect to $C$ axis. Regions IV and $\mathrm{V}$ correspond to the domain where the chains are totally collapsed and demixing occurs (after Daoud and Jannink ref. [6]).

1) Recent progress [8] in the theory of critical phenomena has shown that there are characteristic exponents associated with the cross-over between critical and tricritical behaviour [9].

2) Recent progress in neutron scattering techniques $[10,11,12]$ has allowed a precise observation of the coil pair correlation function, in dilute as well as in semi-dilute solutions, over the entire intermediate
TABLE I

Scaling of the squared end to end distance $\left\langle R^{2}\right\rangle$

\begin{tabular}{ll} 
Region & \multicolumn{1}{c}{$\left\langle R^{2}\right\rangle$} \\
$\overline{\text { I }}$ & $M^{2 v} \tau^{2 / 5}$ \\
I' $^{\prime}$ & $M$ \\
II & $M C^{-1 / 4} \tau^{1 / 4}$ \\
III & $M$ \\
IV & $M^{2 / 3}$
\end{tabular}

$M$ is the molecular weight, $C$ the concentration $\left(\mathrm{g} \mathrm{cm}^{-3}\right)$ and $\tau$ the reduced temperature $\tau=(\tau-\theta) / \theta$. (After Daoud and Jannink [6].)

range of reciprocal space $q$. The variable $q$ is not just one more parameter like $M, T$ or $C$. It in fact bears a precise relation to the cross-over phenomena which helps us to understand a particular aspect of polymer statistics.

The first is currently being studied and will be published elsewhere [13]. We will be concerned here with two experimental observations of the pair correlation function in the temperature-concentration diagram.

2. Remarks on the pair correlation function. - The pair correlation function for a set of $N$ points, representing the monomers of a single polymer, each of which is at a position $\mathbf{r}_{i}(i=1, \ldots, N)$ is defined to be

$$
P(\mathbf{r})=\frac{1}{N^{2}} \sum_{i j}^{N}\left\langle\delta\left(\mathbf{r}-\mathbf{r}_{i}+\mathbf{r}_{j}\right)\right\rangle
$$

where $\langle>$ denotes the average over all configurations. It is customary to consider the second moment of this function, which is related to the average squared radius of gyration $\left\langle R_{\mathrm{G}}^{2}\right\rangle$. However there is more information in (2.1) than in $R_{\mathrm{G}}$ and we shall be particularly interested in the $\mathbf{r}$ dependence of this function. The Fourier transform of $P(\mathbf{r})$

$$
S(\mathbf{q})=\frac{1}{2 \pi} \sum_{i j}^{N-1}\left\langle\mathrm{e}^{i \mathbf{q} \cdot\left(\mathbf{r}_{i}-\mathbf{r}_{j}\right)}\right\rangle
$$

is directly measurable in a scattering experiment. The usual problem associated with (2.2) is the accurate calculation of the inverse Fourier transform from a set of data, which are limited in reciprocal space by the experimental conditions. In the case where the scatterer is a single polymer chain of $N$ segments (we shall denote the scattering law by $S_{1}(\mathbf{q})$ ), there is a more subtle relation between (2.2) and the terms of the sum (2.1), arising from the linear arrangements of the monomers and which can easily be seen from the alternative way of writing (2.2) (valid when edge effects are negligible).

$$
S_{1}(\mathbf{q})=\sum_{n=0}^{N-1}(N-n)\left\langle\cos \mathbf{q} \cdot \mathbf{r}_{n}\right\rangle .
$$


Here the running index $n$ measures the distance between two monomers $(i, j)$ along the chain. It is sometimes called the chemical distance, in contrast to the actual distance $r_{i j}$. As will be shown, the parameter $n$ plays an important role in the understanding of cross-over in polymer solutions. The $q$ dependence of the scattering law $S_{1}(\mathbf{q})$ is determined directly by the scaling of $\left\langle r_{n}^{2}\right\rangle$ with $n$. Suppose that there exists a particular $n$, called $n_{\mathrm{c}}$, such that the scaling below $n_{\mathrm{c}}$ is different from the scaling above $n_{\mathrm{c}}$. We may split (2.3) into two significant terms

$$
S_{1}(\mathbf{q})=S_{1}^{\mathrm{I}}(\mathbf{q})+S_{1}^{\mathrm{II}}(\mathbf{q})
$$

where

$$
\begin{aligned}
S_{1}^{\mathrm{I}}(\mathbf{q}) & =\sum_{n=0}^{n_{\mathrm{c}}-1}(N-n)\left\langle\cos \mathbf{q} \cdot \mathbf{r}_{n}\right\rangle \\
S_{1}^{\mathrm{II}}(\mathbf{q}) & =\sum_{n=n_{\mathrm{c}}}^{N-1}(N-n)\left\langle\cos \mathbf{q} \cdot \mathbf{r}_{n}\right\rangle .
\end{aligned}
$$

Obviously $S_{1}^{\mathrm{I}}$ reflects the scaling below $n_{\mathrm{c}}$, i.e. the correlations of smaller chemical distances. If we consider a value $q$ of reciprocal space in the range

$$
q>\left(\left\langle r_{n_{\mathrm{c}}}^{2}\right\rangle\right)^{-1 / 2}=q^{*}
$$

we see from inspection of (2.4), that the contribution of $S_{1}^{\mathrm{I}}$ to $S_{1}$ is greater than $S_{1}^{\text {II }}$. Conversely, in the range

$$
q<q^{*}
$$

The contribution of $S_{1}^{\mathrm{II}}$ is dominant. Thus from the analysis of $S_{1}(q)$ we are able to derive the behaviour of $\left\langle r_{n}^{2}\right\rangle$ with $n$, as $n$ increases through $n_{\mathrm{c}}$.

There is a large body of evidence supporting the hypothesis that the scaling of $\left\langle r_{n}^{2}\right\rangle$ changes around a characteristic value $n_{\mathrm{c}}$.

1) In a good solvent, in the limit of zero concentration, it is well-known [4] that the excluded volume interaction $v$ swells the coil only if

$$
v / l^{3} \gg N^{-1 / 2} .
$$

This condition expresses in fact the Ginzbourg criterion of the critical phenomena [14]. It can be written in the neighbourhood of the theta temperature $\Theta$ [4]

$$
\tau \gg N^{-1 / 2}
$$

where $\tau$ is the reduced temperature $\tau=(T-\Theta) / \Theta$.

The important step to the understanding of the cross-over phenomena is the extension of condition (2.6) to any chemical distance $n \leqslant N$. At a given temperature $\tau$ for which (2.6) holds, there is a characteristic $n_{\mathrm{c}}$

$$
n_{\mathrm{c}}=\tau^{-2}
$$

such that all distances $\left\langle r_{n}^{2}\right\rangle\left(n \geqslant n_{\mathrm{c}}\right)$ are swollen and all distances $\left\langle r_{n}^{2}\right\rangle\left(n<n_{\mathrm{c}}\right)$ are unperturbed. Defining

$$
\xi_{\mathrm{g}}^{2}=n_{\mathrm{c}} l^{2}
$$

then the mean end-to-end distance is

$$
\left\langle R_{1 N}^{2}\right\rangle=\left(\frac{N}{n_{\mathrm{c}}}\right)^{2 v} \xi_{\mathrm{g}}^{2}
$$

where $v$ is the excluded volume exponent $(v=3 / 5)$. Using (2.7) and (2.8) formula (2.9) yields :

$$
\left\langle R_{1 N}^{2}\right\rangle=N^{6 / 5} \tau_{. .}^{2 / 5} l^{2}
$$

which is a known result $[6,7]$.

2) In a good solvent, but in the semidilute regime, the excluded volume interaction $v$ is screened beyond a characteristic value $n_{\mathrm{cc}}$ by the finite density of coil segments. Here, the distances $\left\langle r_{n}^{2}\right\rangle$ for $n \leqslant n_{\mathrm{cc}}$ are swollen, and the distances $\left\langle r_{n}^{2}\right\rangle$ for $n>n_{\text {cc }}$ are unperturbed, in contrast to the change of behaviour around $n_{\mathrm{c}}$. Previous calculations [15] have shown that $n_{\mathrm{cc}}$ varies with segment concentration $C$ as

$$
n_{\mathrm{cc}} \sim C^{-5 / 4} \text {. }
$$

Defining

$$
\xi_{\mathrm{c}}^{2}=n_{\mathrm{cc}}^{2 v} l^{2}
$$

we have

$$
\left\langle R_{1 N}^{2}\right\rangle=\left(\frac{N}{n_{\mathrm{cc}}}\right) \xi_{\mathrm{c}}^{2},
$$

or by (2.11) and (2.12)

$$
\left\langle R_{1 N}^{2}\right\rangle=N C^{-1 / 4} l^{2}
$$

which was observed in a small-angle neutron scattering experiment [15].

3) Combining 1) and 2) in a semidilute solution we have

$$
n_{\mathrm{cc}} \geqslant n_{\mathrm{c}}
$$

until now, the existence of $n_{\mathrm{c}}$ and $n_{\mathrm{cc}}$ was derived as a theoretical hypothesis, which agrees with the observation of the law (2.10) and (2.14). In the next section we present experimental evidence for the existence of these different behaviours.

3. Experimental study of temperature and concentration cross-overs. - We have investigated by SmallAngle Neutron Scattering cross-overs predicted by the theory [6] in region I (dilute solutions) and region II (semicilute solutions) of the temperature-concentration diagram (Fig. 1). The special features of the scattering technique are amply described in recent papers $[10,11,12]$. All measurements are performed on a small-angle scattering spectrometer of the Laboratoire Léon-Brillouin set on a cold neutron guide of the EL3 reactor at Saclay [10]. The incident 
TABLE II

Samples used in cross-over studies

\begin{tabular}{|c|c|c|c|c|c|c|}
\hline Sample & Polymer & $\begin{array}{l}M_{\mathrm{W}} \\
\times 10^{-5} \\
\end{array}$ & Solvent & $\begin{array}{l}\text { Concentration } \\
\times 10^{2} \mathrm{~g} \mathrm{~cm}^{-3}\end{array}$ & $\begin{array}{c}C^{*} \\
\times 10^{2} \mathrm{~g} \mathrm{~cm}^{-3}\end{array}$ & $\begin{array}{c}q^{*} \\
\times 10^{2} \AA^{-1}\end{array}$ \\
\hline A & PSD & 11 & $\mathrm{CS}_{2}$ & 0.1 & 1.3 & $<1$ \\
\hline B & PSD & 11 & $\mathrm{CS}_{2}$ & 2.5 & 1.3 & 3.59 \\
\hline C & PSD & 5 & $\mathrm{CS}_{2}$ & 4 & 2.5 & 4.94 \\
\hline D & PSD & 5 & $\mathrm{CS}_{2}$ & 15 & 2.5 & $>10$ \\
\hline $\mathrm{E}\left({ }^{a}\right)$ & PSH & 11 & $\mathrm{CS}_{2}$ & 2.5 & 1.3 & $<1$ \\
\hline$F$ & PSH & 11 & $\mathrm{CS}_{2}$ & 4 & 1.3 & $<1$ \\
\hline G & PSH & 11 & $\mathrm{CS}_{2}$ & 7.5 & 1.3 & 1.90 \\
\hline $\mathrm{H}$ & PSH & 11 & $\mathrm{CS}_{2}$ & 10 & 1.3 & 2.44 \\
\hline I & PSH & 11 & $\mathrm{CS}_{2}$ & 25 & 1.3 & 4.78 \\
\hline J & PSH & 11 & $\mathrm{CS}_{2}$ & 50 & 1.3 & $>10$ \\
\hline $\mathrm{K}\left({ }^{b}\right)$ & PSH & 38 & $\mathrm{C}_{8} \mathrm{D}_{8}$ & 0.4 & 4.3 & $\left({ }^{c}\right)$ \\
\hline
\end{tabular}

( $\left.{ }^{a}\right)$ Samples $\mathrm{E}$ to $\mathrm{J}$ contain $0.005 \mathrm{~g} \mathrm{~cm}^{-3}$ of PSD chains of same molecular weight as PSH.

$\left({ }^{b}\right)$ The theta temperature of this system is $38^{\circ} \mathrm{C}$ [27].

$\left({ }^{c}\right)$ See table III

wavelength, defined by a pyrolitic graphite monochromator, is $\lambda=4,70 \pm 0,04 \AA$. Precise definition of angular divergency is achieved by using linear grids and is fixed at a value of $12 \mathrm{~min}$. The scattering vector range set by inequalities (1.1) lies between

$$
10^{-2} \leqslant q \AA^{-1} \leqslant 10^{-1} .
$$

Samples are solutions of polystyrene in different solvents and are listed in table II. The useful signal is obtained by subtracting the scattered intensity of the proper solvent from the scattered intensity of the solution. In the following studies we are concerned only with the $q$ dependence of the scattering functions and not with the precise determination of the constants.

3.1 CONCENTRATION CROSS-OVER. - This crossover is observed in region II of the diagram (Fig. 1), i.e. for solutions in good solvent and in the semidilute range. Two series of experiments were done :

(i) With solutions of deuterated polystyrene (PSD) in carbon disulfide (samples A to D, table II). In this case the scattered intensity is proportional to the Fourier transform of the pair correlation function of any two monomers in the solution. This function will be called $S(q, c)$.

(ii) The contrast factor [16] between protonated polystyrene (PSH) and carbon disulfide is very weak. If few PSD chains are dispersed in this solution, the scattered intensity is then proportional to the Fourier transform of the pair correlation function of two monomers of the same labelled chain $S_{1}(q, c)$. These samples are samples $\mathbf{E}$ to $\mathbf{J}$ listed in table II.

Apart from sample A which is a dilute solution used as a reference in the data interpretation, the concentration in these samples is greater than the overlap concentration $C^{*}$. This concentration is defined as the point for which the different coils, taken as spheres of radius $R_{\mathrm{G}}$, are touching. This condition for a monodisperse solution of polymers of length $N$ is

$$
C^{*} \sim \frac{N}{R_{\mathrm{G}}^{3}} .
$$

Below $C^{*}$ we are dealing with dilute solutions, and the length dependence of $R_{\mathrm{G}}$ is

$$
R_{\mathrm{G}}^{2} \sim N^{6 / 5} l^{2}
$$

using this relation, (3.2) gives

$$
C^{*} \sim N^{-4 / 5} \text {. }
$$

Above $C^{*}$ (semidilute range), the coil formed by $n$ consecutive segments will be expanded by excluded volume interaction if the following inequality holds (see (2.11))

$$
n<n_{\mathrm{cc}} \sim C^{-5 / 4} .
$$

The pair correlation function has been derived by Edwards [5] as

$$
P_{1}(r) \sim r^{-4 / 3}
$$

leading to the Fourier transform

$$
S_{1}(q) \sim q^{-5 / 3} .
$$

For elements with $n$ greater than $n_{\text {cc }}$, screening effects occur and the behaviour of $S_{1}(q)$ is of a random coil type which is simply deduced from the Debye [17] form :

$$
S_{1}(q) \sim q^{-2} .
$$

The existence of the length $\xi_{c}$ defined by equa- 
tion (2.12) means that there is in the reciprocal space a cross-over length $q^{*} \sim \xi_{\mathrm{c}}^{-1}$ with a concentration dependence derived from (2.11) and (2.12)

$$
q^{*} \sim C^{3 / 4} \text {. }
$$

When the concentration varies, the $S_{1}(q, c)$ function takes a random coil form for $q$ less than $q^{*}$ and excluded volume form for $q$ greater than $q^{*}$. In the language of critical phenomena we are dealing with a spatial cross-over.

The expression for $S_{1}(q, c)$ can be obtained by the following argument. For a single chain of $N$ monomers this function is expressed as the first term of a cumulant expansion of (2.2) :

$$
S_{1}(q, c)=\sum_{i j} \exp -\frac{q^{2}}{6}\left\langle r_{i j}^{2}\right\rangle
$$

where $r_{i j}$, the distance between monomers $i$ and $j$, is only a function of the difference $|i-j|$. Neglecting end effects as in (2.2)

$$
S_{1}(q, c)=2 N \sum_{n=1}^{N-1}\left(1-\frac{n}{N}\right) \exp \left(-\frac{q^{2}}{6} r_{n}^{2}\right)+N .
$$

Using the concept of the characteristic length $n_{\mathrm{cc}}(2.11)$, it is possible to split the summation in (3.6) into two parts : one for elements such $n \leqslant n_{\mathrm{cc}}$ where excluded volume effects exist and one for $n_{\mathrm{cc}} \leqslant n \leqslant N$ where these effects vanish :

$$
\begin{aligned}
& S_{1}(q, c)= \\
& \quad=2 N\left\{\sum_{n=1}^{n_{\mathrm{cc}}-1}\left(1-\frac{n}{N}\right) f_{n}+\sum_{n=n_{\mathrm{cc}}}^{N-1}\left(1-\frac{n}{N}\right) g_{n}\right\}+N
\end{aligned}
$$

where

$$
f_{n}=\exp -\frac{q^{2}}{6}\left(\frac{n}{n_{\mathrm{cc}}}\right)^{2 v} \xi_{\mathrm{c}}^{2}
$$

and

$$
g_{n}=\exp -\frac{q^{2}}{6} \frac{n}{N} R_{1 N}^{2}
$$

taking $Z=n_{\mathrm{cc}} / N$ and neglecting the $N$ term in (3.7) a straightforward calculation gives the result

$$
\begin{gathered}
S_{1}(q, c)=N^{2}\left\{\frac{2}{X}\left[\mathrm{e}^{-Z X}(1-Z)-\frac{1}{X}\left(\mathrm{e}^{-Z X}-\mathrm{e}^{-X}\right)\right]+\right. \\
\left.+\frac{Z}{v Y^{1 / 2 v}}\left[\gamma\left(\frac{1}{2 v}, Y\right)-\frac{Z}{Y^{1 / 2 v}} \gamma\left(\frac{1}{v}, Y\right)\right]\right\}
\end{gathered}
$$

where

$$
X=\frac{q^{2} R_{1 N}^{2}}{6}, \quad Y=\frac{q^{2} \xi_{\mathrm{c}}^{2}}{6}
$$

and $\gamma$ are incomplete gamma functions [18]. The formula (3.8) describes the spatial cross-over between random coil form $\left(q<q^{*}\right)$ and excluded volume form $\left(q>q^{*}\right)$. It can be shown that setting $Z=1$ in (3.8) gives the excluded volume form first derived by Weill, Loucheux and Benoit [19]. On the other hand $Z=0$ leads to the Debye formula [17], or the random coil form.

The $S(q, c)$ function can be derived from $S_{1}(q, c)$. As a first step, consider the scattering law $S_{10}(q, c)$ for a single coil without excluded volume interaction. The random phase approximation (RPA) applied [20] to the semidilute solution, with excluded volume interaction gives

$$
S(q, c)=\frac{C S_{10}(q, c)}{1+C v S_{10}(q, c)} .
$$

This expression accounts partially for the observed results. We found however that replacing $S_{10}(q, c)$ by $S_{1}(q, c)$ in the numerator of (3.9) improves the agreement with the data. We have therefore considered the interpolation formula

$$
\begin{aligned}
S(q, c)=C S_{1}(q, c) \frac{1}{1+} & C v S_{10}(q, c) \\
& =C S_{1}(q, c) \frac{q^{2}}{q^{2}+\kappa^{2}} .
\end{aligned}
$$

We found no reference in the literature for the justification of such a substitution. However there are two possible ways leading to formula (3.10). The first approach consists of improving the RPA along the lines of reference [21]. In the second, following reference [22], we consider $S_{1}(q, c)$ in (3.10) as the form factor of the coil and the remaining term as the contribution of the arrangement of the centres of mass. A recent scattering experiment [23] on coils labelled only around the centre confirms qualitatively the behaviour $q^{2} /\left(q^{2}+\kappa^{2}\right)$ (see also ref. [24]).

Results. - The inverse of the intensity scattered by samples $\mathrm{E}$ to $\mathrm{J}$ (see table II) is displayed on figure 2 versus $q^{5 / 3}$. The total concentration appears on the right of each curve. The scattered intensity is due to 0.5 percent of PSD chains and hence corresponds to $S_{1}(q, c)$.

The points are the experimental data and the solid curves represent the result of the calculation by formula (3.8). The agreement is excellent and the theoretical cross-over point is indicated by vertical arrows. For large concentration there is some data scatter, but the behaviour for $q>q^{*}$ is clearly a straight line. Thus it cannot be due to chain stiffness, leading to a downwards curvature in this representation. When the total concentration increases, the behaviour of $S_{1}(q, c)$ changes gradually from an excluded volume form (straight line) to a random coil form (upwards curvature). The cross-over point $q^{*}$ goes from the low to the large value of the scattering vector with a concentration dependence given by (3.5). These $q^{*}$ values are collected in table II. 


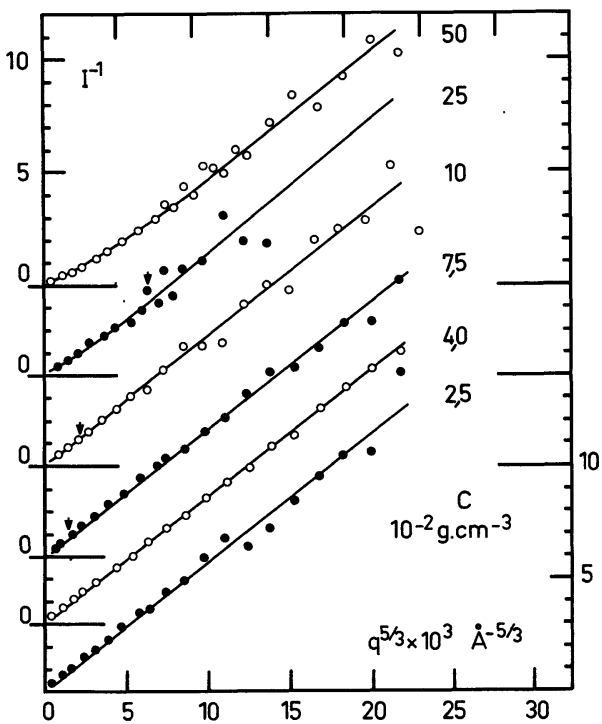

Fig. 2. - Plot of the inverse scattered intensity versus the scattering vector raised to the power $5 / 3$ for samples $E$ to $J$ (see table II). The total concentration $C$ is indicated on the right. Each solution contains a concentration of $0.005 \mathrm{~g} \mathrm{~cm}^{-3}$ of PSD chains. Open and closed points are experimental data. Solid lines are the result of calculation using formula (3.8). Vertical arrows show the theoretical cross-over point.

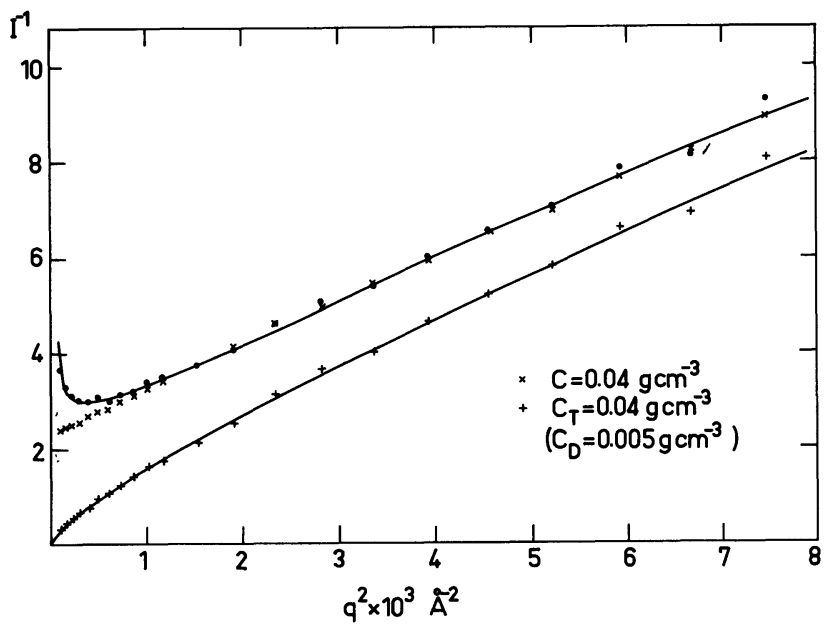

FIG. 3. - Inverse of the scattered intensity versus the squared scattering vector, for sample $C(x)$ and sample $F(+)$. The lower solid curve is calculated by formula (3.8). The upper curve is the result of equation (3.10) when using for $S_{1}(q, c)$ the theoretical result (formula 3.8 ). The solid points are the result when using experimental data of sample $\mathrm{F}$.

Since formula (3.8) gives good agreement with the experimental data, we have used it to calculate $S(q, c)$ by formula (3.10). The result for sample C $\left(C=0.04 \mathrm{~g} \mathrm{~cm}^{-3}\right)$ is the upper curve of the figure 3 , where the inverse of the scattered intensity is plotted versus the squared scattering vector. Measured points are denoted by $x$ and solid curve represents equation (3.10). Closed points are the result of the calculation when using data of sample $\mathrm{F}\left(C_{\mathrm{T}}=0.04 \mathrm{~g} \mathrm{~cm}^{-3}\right)$ in this equation, in place of the theoretical values. For comparison, the lower curve is the theoretical function $S_{1}(q, c)$ (Eq. (3.8)) and crosses are data from sample $F$. This figure shows that the agreement with data of sample $\mathrm{C}$ is good for large $q$ values but there exist disagreements for very low $q$ values. It has been shown [3] that this effect is due to the finite molecular weight.

The behaviour of the $S(q, c)$ function is then not well described by equation (3.10). It can be noted that formula (3.8) giving the $S_{1}(q, c)$ function is the sum of two functions if we use the cross-over point value $q^{*}$

$$
S_{1}(q, c)=F_{\mathrm{A}}\left(q / q^{*}\right)+F_{\mathrm{B}}(q)
$$

this form suggests a better representation of the function by reference to the homogeneous function approach [25].

In the magnetic analogy [26] it has been shown that $S(q, c)$ corresponds to the longitudinal correlation function of the magnetic problem [28]. This function is a homogeneous function of appropriate reduced variables near the critical point [25]. In the polymeric problem the reduced variable will be $q / q^{*}$. In the high concentration range, i.e. for $C$ greater than $C^{*}, S(q, c)$ is a Lorentzian [15]

$$
S(q, c)=\frac{A}{q^{2}+\kappa^{2}}
$$

where $A$ is a constant at a given concentration and $\kappa$ is the inverse of the screening length $\xi$. By reference to this form, the homogeneous function will be

$$
S(q, c)=\frac{1}{q^{2}+\kappa^{2}} F\left(q / q^{*}\right)
$$

where $F(x)$ is the cross-over function. This function is unknown, but behaves asymptotically as

$$
\begin{array}{ll}
F(x) \sim \text { const. } & \text { for } x \ll 1 \\
F(x) \sim x^{1 / 3} & \text { for } \quad x>1 .
\end{array}
$$

The value of $\kappa$ can be extracted from the plot of the inverse scattered intensity versus the squared scattering vector. Then it is possible to plot the function $F(q, c)=\left(q^{2}+\kappa^{2}\right) S(q, c)$ versus $q^{1 / 3}$ where $S(q, c)$ is the actual measured function. According to (3.13) the cross-over will appear in this figure as a plateau followed by a straight line with a positive slope.

Results of this operation are shown on figure 4 for samples A to D (table II). Sample A is the dilute solution for which $S(q, c)$ behaves as $q^{-5 / 3}$ and is used as a reference in this figure. Sample D is a semi-dilute solution and $S(q, c)$ has the lorentzian form (3.11), represented here by a plateau. Between these two extreme behaviours, data for samples $\mathrm{B}$ and $\mathrm{C}$ exhibit the spatial cross-over. The vertical arrows indicate the cross-over point $q^{*}$ defined as the intersection of the asymptotes. This figure shows clearly that $S(q, c)$ is well-described by the homogeneous function (3.12). 


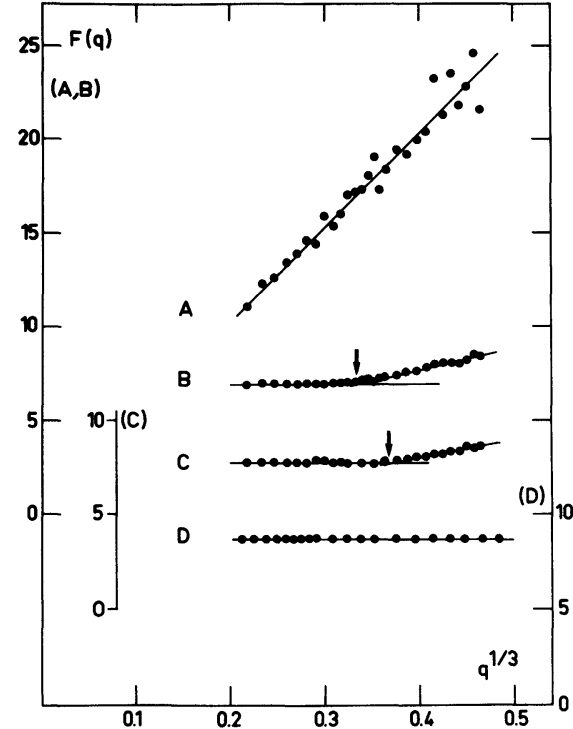

Fig. 4. - Display of the $F(q, c)$ function (eq. (12)) versus $q^{1 / 3}$ for samples A, B, C, and D. Full lines are obtained by a least squares fit method. Vertical arrows indicate the cross-over points. Note the change of origin for samples $C$ and $D$.

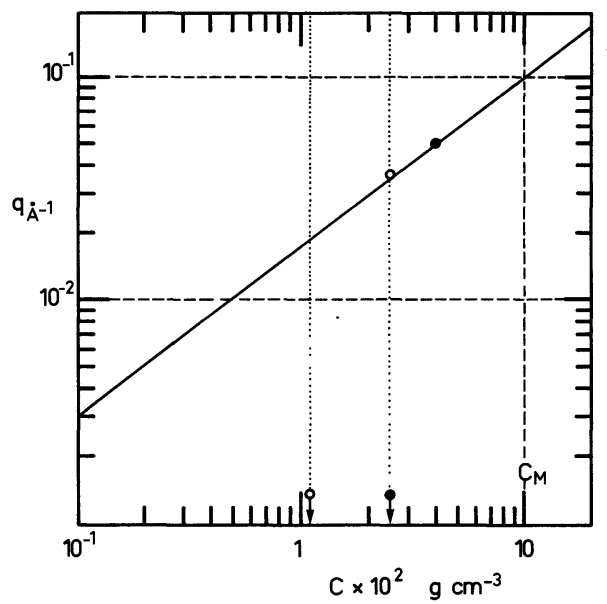

FIG. 5. - Plot of the cross-over point $q^{*}$ versus concentration on a log-log scale. Horizontal dashed lines correspond to the limits of eq. (3.1). Vertical dotted lines are the lower limit for the concentration range, corresponding to the overlap concentration $C^{*}$ (eq. (3.2)). $C_{\mathrm{M}}$ is the maximum of this range given by the intercept of the maximum $q$ value and experimental curve (eq. (3.14)); the two points correspond to experimental values (samples B and C table II)

Experimental values of $q^{*}$ so determined are displayed on figure 5 versus concentration in a $\log \log$ plot. These values agree well with the predicted concentration dependence (3.5). The equation of the straight line is

$$
q^{*}=0.548 C^{3 / 4} .
$$

Experimentally there exist two limits for the observation of this concentration cross-over :

(i) The spatial range given by (3.1) (horizontal dotted lines). (ii) The concentration range limited at the lower limit by $C^{*}$ (vertical dotted line with symbols) and at the upper limit by the maximal concentration $C_{\mathrm{M}}$ given by the intercept of the upper $q$ limit and line (3.14).

Comparison of $q^{*}$ values collected in table II shows that for the same concentration (i.e. samples B and E) the cross-over point of $S(q, c)$ is higher than the cross-over point of $S_{1}(q, c)$.

3.2 TeMPERATURE CROSS-OVER. - Observation of this cross-over was done in region I of the diagram (Fig. 1), i.e. in the dilute range, at constant concentration, by varying the temperature. The sample is a solution of protonated polystyrene ( $\mathrm{PSH}$ ) in deuterated cyclohexane with a concentration ten times lower than $C^{*}$. The characteristics of this sample are collected in table II (sample $\mathrm{K}$ ) the $\theta$ point of this solution is $38^{\circ} \mathrm{C}$ [27]. The solvent used in the subtraction procedure is a mixture of deuterated and protonated cyclohexane in order to compense the large incoherent background due to the protons of PSH. Sample holder and temperature control are described elsewhere [7]. Measurements of sample temperature are obtained with an accuracy of $\pm 0.01{ }^{\circ} \mathrm{C}$ and the regulation is within $\pm 0.05^{\circ} \mathrm{C}$.

In this domain of the diagram, we are dealing with single chains and excluded volume effects are important. In this case the characteristic length $n_{\mathrm{c}}(2.7)$ is a boundary between a critical behaviour for elements $n>n_{\mathrm{c}}$ and a random coil behaviour for $n<n_{\mathrm{c}}$. The description used in part 3.1 applies, but in reverse. The temperature dependence of the characteristic length $\xi_{\mathrm{g}}(2.8)$ is derived from (2.7) leading to a cross-over point varying as

$$
q^{*} \sim \tau \text {. }
$$

The correlation function of one single chain can also be expressed by the same equation as (3.7)

$$
\begin{aligned}
& S_{1}(q, \tau)= \\
& =2 N\left\{\sum_{n=1}^{n_{\mathrm{c}}-1}\left(1-\frac{n}{N}\right) f_{n}+\sum_{n=n_{\mathrm{c}}}^{N-1}\left(1-\frac{n}{N}\right) g_{n}\right\}+N
\end{aligned}
$$

with the new definition of functions $f_{n}$ and $g_{n}$

$$
\begin{aligned}
& f_{n}=\exp -\left(\frac{q^{2}}{6} \frac{n}{N} R_{1 N}^{2}\right) \\
& g_{n}=\exp -\left(\frac{q^{2}}{6}\left(\frac{n}{n_{\mathrm{c}}}\right)^{2 v} \xi_{\mathrm{g}}^{2}\right)
\end{aligned}
$$

$R_{1 N}^{2}$ being now defined by (2.9). Setting $Z=n_{\mathrm{c}} / N$, the calculation gives

$$
\begin{gathered}
S_{1}(q, \tau)=N^{2}\left\{\frac{2 Z}{Y^{2}}\left(Y-Z+\mathrm{e}^{-Y}(Z(1+Y)-Y)\right)+\right. \\
+\frac{1}{v X^{1 / 2 v}}\left[\left(\gamma\left(\frac{1}{2 v}, X\right)-\gamma\left(\frac{1}{2 v}, X Z^{2 v}\right)\right)\right. \\
\left.\left.-\frac{1}{X^{1 / 2 v}}\left(\gamma\left(\frac{1}{v}, X\right)-\gamma\left(\frac{1}{v}, X Z^{2 v}\right)\right)\right]\right\}
\end{gathered}
$$


where $X, Y$ and $\gamma$ have the same definition as in (3.8). Equation (3.16) describes the temperature cross-over of $S_{1}(q, \tau)$. It is easy to check that in the high temperature limit $(Z=0)$, this equation takes the excluded volume form [19]. On the other hand, at the limit of region $\mathrm{I}$, given by $\tau \sim N^{-1 / 2}$ between the dilute regime and the theta solvent range the polymeric chain behaves as a random coil $(Z=1):(3.16)$ reduces to the Debye formula.

An example of scattered intensity by dilute solution (sample $\mathrm{K}$ ) is shown on figure 6 for two temperatures $T_{1}=67.2^{\circ} \mathrm{C}$ and $T_{2}=41.25^{\circ} \mathrm{C}$ corresponding respectively to the reduced temperatures $\tau_{1}=0.0948$

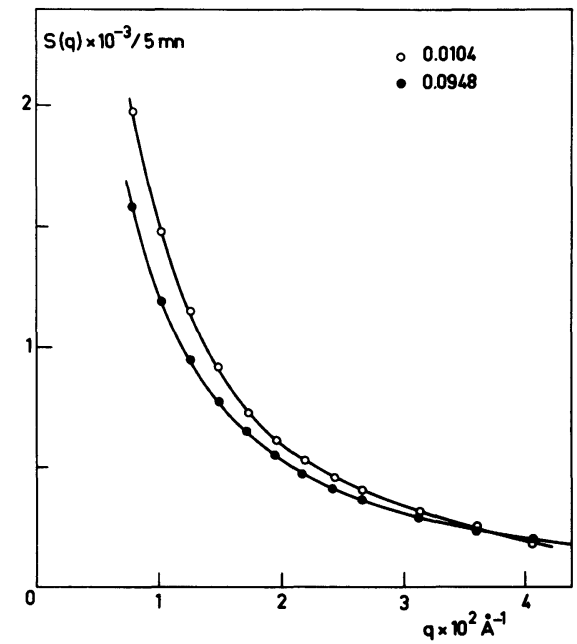

FIG. 6. - Intensity distribution for sample $\mathrm{K}$ (Table II) versus scattering vector $q$ obtained for two temperatures : open circle $41.25^{\circ} \mathrm{C}(\tau=0.0104)$, closed circle $67.2^{\circ} \mathrm{C}(\tau=0.0948)$.

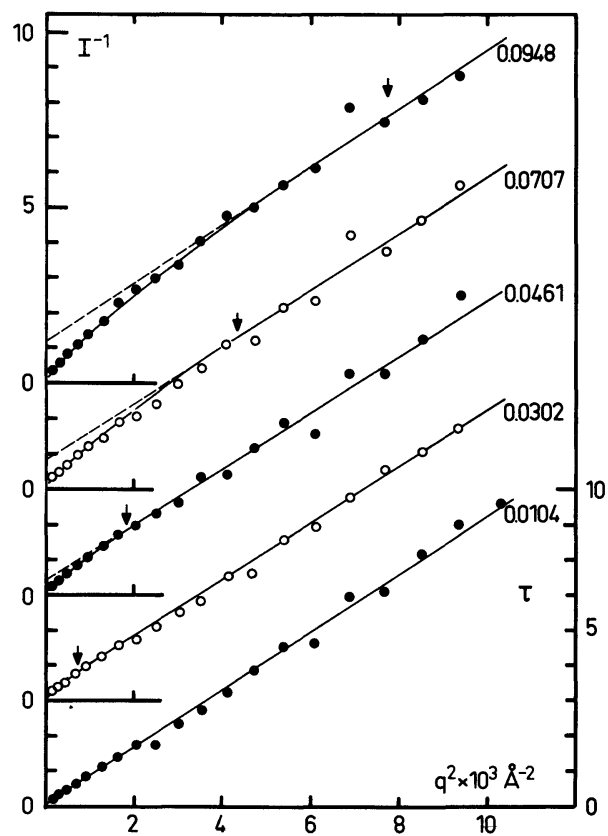

Fig. 7. - Inverse of the scattered intensity versus the square of the scattering vector. Points are experimental data recorded at different reduced temperatures $\tau$ as indicated on the right. The solid curves are the results of calculation using the formula (3.16). Vertical arrows show the theoretical cross-over point.

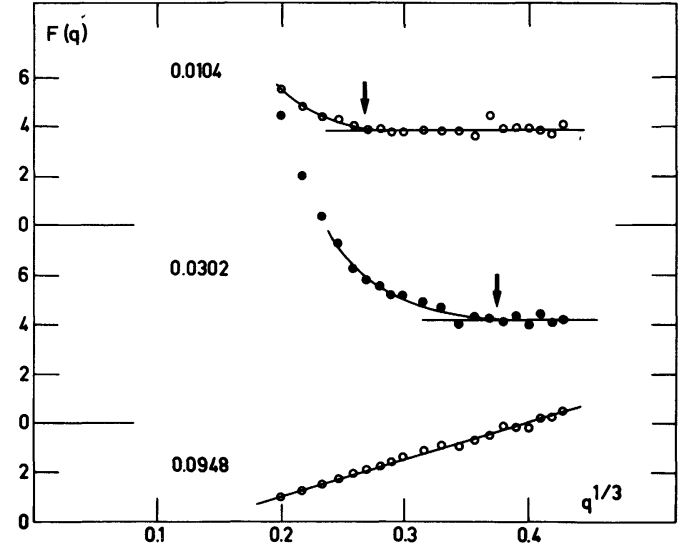

FIG. 8. - Homogeneous function (see text section 3) versus $q^{1 / 3}$ for three different reduced temperatures (indicated on the left). The cross-over point is less well-defined than in figure 4 due to the discontinuity near $q=0$. Vertical arrows indicate experimental cross-over points.

TABLE III

Temperature cross-over point values

\begin{tabular}{|c|c|c|c|}
\hline$T$ & & \multicolumn{2}{|c|}{$q^{*} \AA^{-1} \times 10^{2}$} \\
\hline${ }^{\circ} \mathrm{C}$ & $\tau \times 10^{2}$ & $\exp$ & theo \\
\hline - & - & - & - \\
\hline 41.25 & 10.4 & 2.01 & $<1$ \\
\hline 42.50 & 1.45 & 2.70 & $<$ \\
\hline 43.75 & 1.85 & 3.46 & $<1$ \\
\hline 46.20 & 2.64 & 4.51 & 2.08 \\
\hline 47.40 & 3.02 & 5.27 & 50 \\
\hline 52.30 & 4.61 & $>10$ & 0 \\
\hline 59.70 & 7.07 & $>10$ & .50 \\
\hline 67.20 & 9.48 & $>10$ & \\
\hline
\end{tabular}

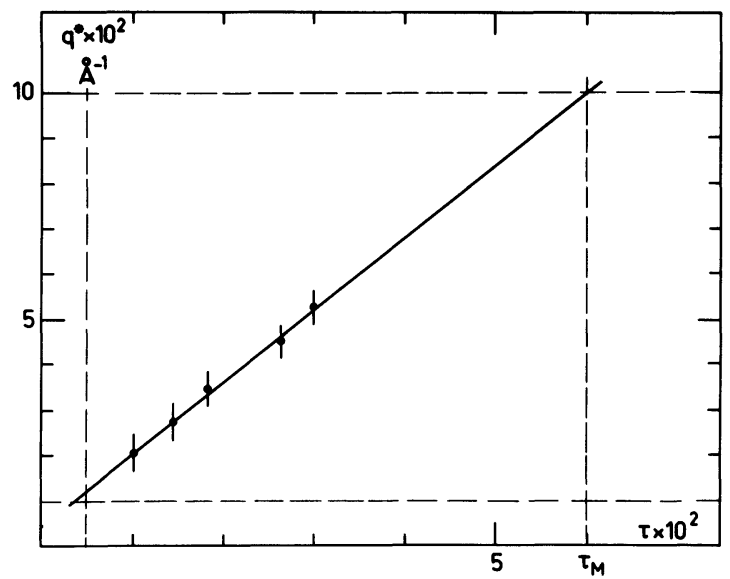

Fig. 9. - Plot of the temperature cross-over point versus reduced temperature $\tau$. Horizontal dashed lines indicate limits of the observation range given by $10^{-2} \leqslant q \AA^{-1} \leqslant 10^{-1}$. The lower vertical dashed lines correspond to the condition (2.6). $\tau_{M}$ is the maximum value corresponding to the intercept between maximum value of $q^{*}$ and experimental curve (eq. (3.17)). 
and $\tau_{2}=0.0104$. One can see from this figure that the scattered intensity increases with decreasing temperature at low $q$ values but is temperature independent at large values. The inverse of the scattered intensity plotted versus $q^{2}$ is displayed in figure 7 for different temperatures between $\tau=0.0104$ and $\tau=0.0948$ as indicated on the right of this figure. Full lines are theoretical calculation (equation (3.16)) normalized to experimental data. Vertical arrows indicate the theoretical cross-over points. It is difficult to define this point precisely with the data displayed on figure 7 , so we tried to use the homogeneous function description in order to obtain experimental cross-over point values. Using equation (3.12) with $\kappa^{2}$ values extrapolated from figure 7 we have plotted the function $F(q)$ versus $q^{1 / 3}$. An example of these curves for three temperatures is given on figure 8 . The typical features as revealed by figure 4 are present but with an important modification. At high temperature the function $S_{1}(q, \tau)$ varies as $q^{5 / 3}$ but in lowering the temperature there is a departure from this behaviour : the random coil behaviour appears at large $q$ values and gradually invades all the range of observation. The cross-over point, indicated by vertical arrows, goes from the right to the left of the figure when the temperature goes down. But as the critical behaviour is in the low $q$-value range, the application of formula (3.12) introduces a discontinuity for $q=0$, giving the curved part of the distribution. The cross-over point is given by the end of the plateau and the values obtained for different temperatures are listed in (Table III) together with the theoretical values obtained from equation (3.16). The comparison between these values shows that the experimental cross-over point values are greater than the theoretical ones. This is not surprising because the theoretical model requires that $q^{*}=0$ for $\tau=N^{-1 / 2}$. Experimental values of the cross-over points are displayed versus $\tau$ values on figure 9 . The data so obtained agree well with a linear variation with $\tau(3.15)$ and a least-squares method gives the following equation

$$
q^{*}=1.606 \tau+0.38 \times 10^{-2}
$$

where $q^{*}$ is expressed in inverse angström and $\tau$ is the reduced temperature. The range of observation of this cross-over is also indicated as in figure 5 by dotted lines.
4. Conclusion. - The existence of characteristic chemical lengths $n_{\mathrm{c}}$ and $n_{\mathrm{cc}}$ is inferred from the observed momentum transfer dependence of the scattering law $S_{1}(q)$. The terminology relevant to the diagram of figure 1 can be used to describe the effective distances along the coil. As $n$ decreases through $n_{\mathrm{c}}$, the behaviour changes from critical to tricritical. As $n$ increases through $n_{\mathrm{cc}}$ the behaviour changes from critical to a mean field type. Further comments are as follows.

1) The observation of the polymer coil configuration provides a unique example of spatial cross-over, i.e. a change of behaviour which is controlled by the chemical distance $n$.

2) The polymer coil configuration is usually characterized by the squared radius of gyration $R_{\mathrm{G}}^{2}$ and the squared step length $l^{2}$. However, if excluded volume effects are to be accounted for properly, it is necessary to include the characteristic correlation length $\xi_{\mathrm{g}}^{2}(2.8)$ and $\xi_{\mathrm{c}}^{2}(2.12)$. These lengths are sometimes used to partition the coil into so-called blobs [13]. The blob concept helps to visualize the effects described above; it is however misleading to consider the coil as a succession of blobs, since the fundamental quantity is the chemical distance between segments and not their position.

3) The concept of $n_{c}$ and $n_{\mathrm{cc}}$ reconciles the proposition that there can be only one value for the critical exponent $v$, with the observation of the scaling law of $R_{G}^{2}$ as a function of molecular weight. The appearance of intermediate values of the exponent between $v=3 / 5$ and $v=1 / 2$ can be resolved in the following expression :

$$
\begin{aligned}
R_{\mathrm{G}}^{2} & =l^{2}\left(N Z^{2}\left(\frac{1}{2}-\frac{Z}{3}\right)\right)+ \\
& +N^{2 v}\left\{\frac{1}{1+2 v}\left(1-Z^{2 v+1}\right)-\frac{1}{2 v+2}\left(1-Z^{2 v+2}\right)\right\}
\end{aligned}
$$

where $Z=n_{\mathrm{c}} / N$.

Acknowledgments. - We wish to thank our colleagues of the CRM Strasbourg for their help in this work. We are grateful to Pr. J. L. Lebowitz for his comments about the manuscript. We thank Prs. J. M. Deutch and H. Boots for stimulating questions about this subject.

\section{References}

[1] KIRSTE, R. G., in Proc. Conf. on Small Angle X Ray Scattering Syracuse, USA (1965) H. Brumberger ed.

[2] Farnoux, B., Daoud, M., Decker, D., Jannink, G. and OBER, R., J. Physique Lett. 36 (1975) L-35.

[3] Far noux, B., Ann. Phys. I (1976) 73.

[4] FloRy, P. J., Principles of Polymer Chemistry (Cornell University Press 6th ed.) 1966
[5] Edwards, S. F., Proc. Phys. Soc. 85 (1965) 613.

[6] DaOUd, M. and Jannink, G., J. Physique 37 (1976) 973.

[7] Cotton, J. P., Nierlich, M., Bout, F., Daoud, M., Farnoux, B., Jannink, G., Duplessix, R. and Picot, C., J. Chem. Phys. 65 (1976) 1101.

[8] De Gennes, P. G., J. Physique Lett. 36 (1975) L-55.

[9] FisHer, M. E., Rev. Mod. Phys. 46 (1974) 597. 
[10] Cotton, J. P., Decker, D., Benoit, H., Farnoux, B., Higgins, J., Jannink, G., Ober, R., Picot, C. and Des CloIZEAUX, J., Macromolecules 7 (1974) 863.

[11] Schmatz, W., Springer, T., Schelten, J. and Ibel, K., J. Appl. Crystallogr. 7 (1974) 96.

[12] Kirste, R. G., Kruse, W. A., Ibel, K., Polymer 16 (1975) 120.

[13] Dạud, M., Thesis, Université de Paris (1977).

[14] Toulouse, G. and Pfeuty, P., Introduction au groupe de renormalisation et à ses applications (Presses Universitaires, Grenoble) 1975.

[15] Daoud, M., Cotton, J. P., Farnoux, B., Jannink, G., Sarma, G., Benoit, H., Duplessix, R., Picot, C. and DE GeNNES, P. G., Macromolecules 8 (1975) 804.

[16] Cotton, J. P. and Benort, H., J. Physique 36 (1975) 905.

[17] Debye, P., J. Phys. Colloid. Chem. 51 (1947) 18.

[18] Table of Integrals, series and products, I. S. Gradshteyn, I. M. Ruzhik ed. (Academic Press New York) 1965.
[19] Loucheux, C., Weill, G. and Benort, H., J. Chim. Phys. 43 (1958) 540

[20] Jannink, G. and De Gennes, P. G., J. Chem. Phys. 48 (1968) 2260.

[21] Nelkin, M., Phys. Rev. 183 (1969) 349.

[22] GUinier, A. and Fournet, G., Small-Angle Scattering of $X$ rays (Wiley, New York) 1955.

[23] Cotton, J. P., to be submitted.

[24] De Gennes, P. G., J. Physique 31 (1970) 235.

[25] Stanley, E., Phase transitions and critical phenomena (Oxford Press) 1971, chap. 11.

[26] Des Cloizeaux, J., J. Physique 36 (1975) 281.

[27] Strazielle, C. and Benort, H., Macromolecules 8 (1975) 203.

[28] SCHÄFeR, L. and Witten, T. A., J. Chem. Phys. 66 (1977) 2121.

[29] BurCh, D. J. and Moore, M. A., J. Phys. A 9 (1976) 451.

[30] MOORE, M. A., J. Physique 38(1977) 265. 